\title{
Health education and leprosy
}

\author{
CME MATTHEWS, ${ }^{*}$ AJ SELVAPANDIAN \& \\ M JESUDASAN \\ Departments of Community Health and Orthopaedics and \\ Leprosy Reconstructive Surgery - CMC Hospital, Vellore
}

Received for publication 14 October 1979.

\begin{abstract}
Summary In the context of effective health education in leprosy, various theories from the behavioural sciences are reviewed. In a project carried our near Vellore in South India over a period of $1 \frac{1}{2}$ years, the three main stages included information, motivation and action. The objectives were to make the patients come for early and regular treatment, to make the public willing to employ patients and not to avoid harmless contact with them, and to make patients take proper care of their hands and feet. The techniques employed are described and the results analysed indicating a considerable improvement in knowledge, attitude and reported practice.
\end{abstract}

The aim of health education is to change people's behaviour in a way that is beneficial for their health. Merely giving information is seldom adequate to achieve this change in behaviour, and a learning experience is usually more effective. However this is difficult in leprosy because no quick results can be seen. Health education is based on the behavioural sciences - social psychology, sociology and anthropology, and applies insights from these sciences to bring about behaviour change. It is important to understand the beliefs of the people before starting to give health education, and here anthropological studies are of value. For example many people do not connect the patch with leprosy at all; they call it 'themal' in Tamil, and think it is the same as tinea, or they think it is a bite and do not take it seriously. The name 'leprosy' is reserved for the advanced disease with deformities. Thus to ask a person with an apparently harmless patch to attend a clinic where he will have to mix with advanced leprosy patients and to carry the same stigma as they do, is to make cooperation unnecessarily difficult. If patients with a patch were referred to a general clinic

\footnotetext{
*Present address: YMCA Tribal Development Project, Yellagiri Hills, Tamil Nadu, S. India, 635853.
} 
then the response might be much improved. Bhat $(1978)^{1}$ and colleagues in Karnataka told patients with a patch that their disease would develop into leprosy if they did not take treatment (which is true in terms of their concepts). In this way they were able to persuade patients to take treatment which they would not have agreed to if they had been told that they had leprosy.

Various theories from the behavioural sciences can be applied in health education. We have carried out a project in 5 panchayats near Vellore (Matthews, Jesudasan, Selvapandian and Benjamin 1979;2 Matthews and Jesudasan $1978 ;^{3}$ Jesudasan and Matthews 1978), ${ }^{4}$ based mainly on the theories of Cartwright $(1949)^{5}$ and Lionberger (1960), ${ }^{6}$ as well as a combination of several theories formulated by Matthews (1975) $;^{7}$ see also Matthews, Benjamin, Samikkannu, Punithavithy and Palocaren (1977). ${ }^{8}$ According to Cartwright's theory, it is necessary to change cognitive, motivational and action structure in order to change behaviour. To change cognitive structure, new information must be presented to the people in a form which is clear and acceptable to them. To change motivational structure the required behaviour must be seen as a path to some goal the person has. To provide the right action structure the person must have an easily available opportunity to carry out some specific action to produce the required behaviour.

Lionberger postulates five stages in the adoption of an innovation: 1. Awareness, 2. Interest, 3. Evaluation, 4. Trial, and 5. Adoption. For the first two stages the mass media can be effective, but for the later stages personal influence is more important, and for final adoption personal experience is what matters most.

According to Matthews' theory the probability that an action will be taken depends on the product of three factors:

1. The perceived probability that the action will lead to the goal $(p)$,

2. The perceived importance of the goal $(i)$,

3. The perceived effort required $(e)$.

Since probability of taking action increases with decreasing effort, the third factor is $(1-e)$ and not $e$. The product is therefore $i p(1-e)$. Thus for advanced leprosy patients the goal of curing their disease may be of great importance to them (high $i$ ), but they may not believe in allopathic treatment (low $p$ ), and effort in taking treatment for so long may be great (high $e$ ). Therefore the product $i p(1-e)$ will be low and the action (taking treatment) may not be taken. For patients with only a patch, the problem will be even more difficult as already discussed. The effort $(e)$ involved in taking treatment will be high due to the stigma and the importance of the goal will be low since the goal of curing the patch is not taken very seriously.

In the project carried out near Vellore (Matthews et al., 1979), ${ }^{2}$ there were three stages of education during $1 \frac{1}{2}$ years - information, motivation and action corresponding to $p, i$, and $(1-e)$. The objectives were to make patients come 
for early and regular treatment, to make the public willing to employ patients and not to avoid harmless contact with them, and to make patients take proper care of their hands and feet. An intermediate objective was to improve the knowledge of and attitude towards leprosy of patients and general public so as to achieve the required change in behaviour.

A knowledge, attitude and practice survey was carried out before and after the education was given, for evaluation. The detailed results of this survey before education was given have already been published (Matthews and Jesudasan 1978). ${ }^{3}$ The information stage included training of staff, training camps for village leaders, public meetings, drama, film shows, leaflets, slides in

Table 1. Knowledge

\begin{tabular}{|c|c|c|c|c|}
\hline & \multicolumn{4}{|c|}{$\%$ Respondents } \\
\hline & \multicolumn{2}{|c|}{ General public } & \multicolumn{2}{|c|}{ Patients } \\
\hline & Before & After & Before & After \\
\hline \multicolumn{5}{|l|}{ Knowledge of: } \\
\hline Patch as early sign & 21 & 70 & 54 & 91 \\
\hline Cause due to germs & 0 & 28 & 1 & 28 \\
\hline Complete cure possible & 52 & 85 & 73 & 95 \\
\hline Not all types infectious & 0 & 31 & 16 & 47 \\
\hline Incubation time more than 3 yrs & 17 & 82 & 22 & 84 \\
\hline Prevention by treatment and check up & 34 & 64 & 30 & 64 \\
\hline Injuries due to anaesthesia & \multicolumn{2}{|c|}{ Not asked } & 6 & 20 \\
\hline \multicolumn{5}{|l|}{ Patients without deformities can } \\
\hline do hard work/all work & 70 & 91 & 65 & 96 \\
\hline \multicolumn{5}{|l|}{ Patients with deformities can do } \\
\hline light work & 19 & 36 & 27 & 38 \\
\hline
\end{tabular}

Table 2. Attitude

\begin{tabular}{ccccccc}
\hline & \multicolumn{2}{c}{ General public } & & \multicolumn{2}{c}{ Patients } \\
\cline { 2 - 3 } \cline { 6 - 7 } & Before & After & & Before & After \\
\hline Mean score $(\%)$ & -12 & 43 & & 3 & 50 \\
\hline
\end{tabular}

cinemas, posters, and talks with flash cards to small groups. At the motivation stage various group meetings were held involving leaders, women, men, youth, teachers, etc. Leaders and regular patients were used to motivate those not taking regular treatment. Groups of patients were educated separately with emphasis on care of hands and feet. In the action stage attempts were made to remove barriers to action by reminding defaulters about the clinic, giving treatment by proxy for those who could not come, and in one village by running the clinic as a general skin clinic.

The results of the knowledge, attitude, practice survey after the three stages of education showed a considerable improvement in knowledge, attitude and 
Table 3. Practice (patients only)

\begin{tabular}{lcc}
\hline & \multicolumn{2}{c}{$\%$ Respondents } \\
\cline { 2 - 3 } & Before & After \\
\hline Go to hospital for dressing injury & 69 & 95 \\
Wear chappals always & 17 & 34 \\
Take care when cooking & 9 & 36 \\
Take advice from doctors, nurses, PMWs & 21 & 79 \\
\hline
\end{tabular}

reported practice. However some follow up is required to assess fully changes in practice. Some of the more important improvements are shown in Tables, 1, 2 and 3. The full results are given in the original report (Matthews et al., 1979), ${ }^{2}$ and the results before education in a previous publication (Matthews and Jesudasan 1978). ${ }^{3}$ It can be seen from Tables $1-3$ that there is a considerable effect. After the education many more respondents recognize the patch as an early sign of leprosy, and most know now that complete cure is possible. Before, practically none knew the cause of leprosy, now at least some know. Improvement is also seen in knowledge of infectivity, incubation time, prevention, cause of injuries, and patients' ability to work. Attitude (measured by Likert scale) has changed from being negative or only slightly postive to being much more strongly positive (Table 2.). After the education more patients say that they wear sandals always, go to hospital for dressing of injuries, take care when cooking, and take advice from doctors, nurses or paramedical workers.

The minimum resources required to produce such an effect in a population of about 200,000 would include, in addition to the usual staff of a leprosy control unit, one health educator, one social worker, and (for 6 months only) 2 projectionists and 2 projectors. The project would require a minimum of 2 years, preferably 3 years. A detailed plan has been made for such a project.

In another study (Matthews and Benjamin 1979), ${ }^{9}$ it was found that even after many years of teaching individuals through home visits, little affect had been obtained, whereas the present project has produced considerable affect after only $1 \frac{1}{2}$ years of education. In this other study the service area (population 7,560) was compared with a control area (population 7,260) in the same block. A knowledge, attitude and practice survey was carried out on a random sample in each area (sample sizes 289 and 284 in service and control areas respectively). Another similar survey in 2 different blocks was also carried out; the populations were 84,000 and 90,000 and sample sizes 1,528 and 1,580 in these two blocks. Attitudes, measured in a similar way to that used in the present study were $0.2 \%$ in the service area and $5.3 \%$ in the control area of the first block. In the other two blocks attitudes were $-0.3 \%$ and $20.0 \%$. These differences were not significant except for the 
value of $20.0 \%$ which was attributed to a special leprosy project in that area carried out by SLRT Centre, Karigiri, for 13 years up to the date of the study. Knowledge of symptoms and cause was not significantly different in any of the areas, even in the Karigiri project area, and was similar to that found in the present study before the education. Symptoms mentioned were mostly deformities and ulcers, with only about $10 \%$ mentioning the patch. Cause was believed to be infection (by 8 to 13\%), heredity or excessive sexual intercourse, with no significant differences between different areas.

Thus the comparison of the present study with this other one seems to indicate that the application of behavioural science principles does give a real improvement in results.

\section{Acknowledgements}

We are grateful to Dr V Benjamin, Professor and Head of the Department of Community Health for his guidance, also to Dr Jeyaprakash Muliyil and other staff of Rural Health Centre for their help. We also thank Mr P. Kuppusamy, Social Welfare Officer, Department of Orthopaedics and Leprosy Reconstructive Surgery for much help, as well as the field workers Mr Perumal Das, Miss Sirujunisa and Miss Stella Mary.

\section{References}

1 Bhat EN. Socio-cultural aspects of leprosy eradication in Bharwar District. Paper read at Xth International Congress of Anthropological and Ethnological Sciences, Postplenary session on Medical Anthropology, Poona, December 1978.

2 Matthews CME, Jesudasan M, Selvapandian A, Benjamin V. Health education in leprosy, 1979 (in press).

3 Matthews CME, Jesudasan M. A leprosy health education project. Internat J Leprosy, $1978,46,414$.

4 Jesudasan M, Matthews CME. Involvement of community leaders in leprosy control leaders training camps - an evaluation study. Rehabilitation in Asia, 1978, 19, No 3, 24.

5 Cartwright D. Some principles of mass persuasion. Human Relations, 1949, 2, 253.

6 Lionberger HF. Adoption of new ideas and practices. Iowa State University Press, USA, 1960.

7 Matthews CME. A theory for predicting behaviour change. Internat J Hlth Ed, 1975, Supplement to 18 , No 2 .

8 Matthews CME, Benjamin V, Samikkannu KC, Punithavithy D, Palocaren A. Education to overcome malnutrition in rural preschool children. Internat J Hlth Ed. 1977, Supplement to 30 , No 4 .

9 Matthews CME, Benjamin V. Health education evaluation and beliefs and practices in rural Tamil Nadu, Social Action, 1979, 29, 115. 